\title{
Exact Density of States for finite Gaussian Random Matrix Ensembles via Supersymmetry
}

\author{
Frieder Kalisch* and Daniel Braak \\ Institut für theoretische Physik II, Universität Augsburg, \\ 86135 Augsburg, Germany \\ (Dated: January 31, 2002)
}

\begin{abstract}
We calculate the exact density of states (DOS) for the three classical and two non-classical Random Matrix Ensembles for finite matrix size $N$ using supersymmetric integrals. The $1 / N-$ Expansion yields already in lowest order good approximations to the exact result even for small values of $N \sim 5$. We conjecture a connection between the $N$-dependence of the oscillating part of the DOS and the short-distance behavior of the two-level correlation function.

PACS numbers: 02.50.Ey,05.45.+b, 21.10.-k, 24.60.Lz, 72.80.Ng
\end{abstract}

\section{INTRODUCTION}

Random Matrix Theory (RMT) has become a wellestablished tool to describe the statistical properties of the energy levels of quantum many-particle systems in the ergodic regimel. It is based on the assumption that (apart from global symmetry conditions as spin rotationor time reversal invariance) the Hamiltonian $H$ of a system with $N$ states is described by a probability distribution

$$
\left\langle H_{i j}\right\rangle=0, \quad\left\langle H_{i j} H_{k l}^{*}\right\rangle=g \delta_{i k} \delta_{j l} .
$$

RMT derives the statistical properties of the eigenvalues of $H$ from this distribution of its matrix elements. Whereas most of the applications are still centered around the three classical Wigner-Dyson (WD) Ensembles, the Gaussian unitary, orthogonal and symplectic ensembles (GUE, GOE and GSE), there is also interest in certain generalizations of the standard cases, leading to seven additional symmetry classes, which differ from each other by their behavior under a set of discrete spacetime transformations. Three of them are the chiral analogues to the WD-Ensembles and four are relevant for mesoscopic normal-superconducting hybrid systemse.

RMT is a soluble description of the statistics of disordered and chaotic systems in the sense that all $n$-level correlation functions can be computed in principle exactly. The most important of these correlation functions is the two-level correlation function as it contains already the main information about the repulsion of neighboring levels and discerns the three WD-Ensembles unambiguously. Several mathematical tools are available to compute these correlation functions, e.g. the method of or thogonal polynomials 3 , or the sypersymmetric method 1 . The last method maps the matrix ensemble to a zerodimensional non-linear sigma-model in the limit of large matrix size $N$. Because then the limit $N \rightarrow \infty$ is implicit in all calculations, it is not possible to study directly

*e-mail: frieder.kalisch@physik.uni-augsburg.de finite-size effects, i.e. the dependence of the average level spacing $\Delta(E)$ at energy $E$ on $N$.

Instead one is forced to fix a certain scaling of the overall normalization of $H$ with $N$. In this way the two energy scales, the bandwidth $2 E_{\max }$ and the mean level spacing $\Delta(E)$, are entangled with the matrix size. Either the bandwidth is kept at a fixed finite value (macroscopic scaling) or the mean level spacing $\Delta(E) \sim 1$ at a certain energy $E$ (microscopic scaling). The first scaling is used for the DOS (and yields the semi-circle for $N \rightarrow \infty$ ), the second is appropriate to extract the universal level repulsion. The non-classical Ensembles (we will treat class D and Class $\mathrm{C}$ in this article) show characteristic features of the DOS, deviating from the classical ensembles, but this was calculated only in the microscopic scaling limit.20.6 The DOS varies strongly on the scale of the level spacing for $E \sim 0$, and becomes therefore dependent on the position within the spectrum. In this case the usual unfolding procedure which normalizes the DOS to a constant over many consecutive levels is inapplicable. The question arises, whether the new features of the DOS survive the macroscopic scaling $\Delta(E) \sim 1 / N$ leading to a finite band width, which is the physical relevant case for solid state applications. It seems therefore advisable to compute the DOS exactly for finite $N$ and to study its behavior in the limit $N \rightarrow \infty$ using this formula. The only results for finite $N$ were so far obtained via the orthogonal polynomial method. We will show in the following that the supersymmetric method can be used as well while being technically simpler and more versatile.

The outline of the paper is as follows: In section II we study the simplest case, the GUE, and explain in detail how the exact DOS for finite $N$ can be obtained. We then use a saddle-point method to derive a $1 / N$-expansion. This expansion is not based on a supersymmetric saddlepoint manifold but to the contrary is effected by exact integration over the fermionic variables, which inevitably breaks explicit supersymmetry. Taking into account the quadratic fluctuations of the remaining bosonic variables yields then already excellent approximations to the exact result for small $N$ and everywhere in the spectrum except at the band edge, where the $1 / N$-expansion diverges. In Section III we give the exact DOS for finite 
$N$ of the orthogonal and symplectic ensembles as well as class $\mathrm{C}$ and D. These ensembles are especially simple among the non-classical ones because the joint eigenvalue distribution can be associated with free fermions on a line in an external potentiall. Nevertheless the calculations of the DOS become only simple if the confining part of the potential is neglected, corresponding to microscopic scaling, thereby loosing all information about the relation between $\Delta(E)$ and $N$ which is just given by the confinement. Therefore we treat Class $\mathrm{C} / \mathrm{D}$ here together with the WD-Ensembles. Regarding the level repulsion they belong to the unitary universality class, i.e. without time reversal invariance (see below). Our method, of course, extends to all other Gaussian Ensembles as well. Section IV contains the $1 / N$-expansion around the bosonic saddle-points for GOE, GSE and Class C/D. We observe a relation between the $N$-dependence of the oscillatory part of the correction terms and the short distance behavior of the two-level correlation function. Then we determine the average level spacing in the band center, where the DOS of class C and D deviates strongly from the (constant) behavior known from the WD-Ensembles, in a way independent from any scaling assumption. Section $\mathrm{V}$ contains a summary of our results.

\section{THE UNITARY ENSEMBLE}

The ensemble consists of complex hermitian $N \times N$ Matrices $H=H^{\dagger}$. We represent the DOS at complex energy $z(\operatorname{Im} z>0)$ as the following expectation value (summation convention is understood):

$$
\langle n(z)\rangle=\frac{1}{\pi N} \operatorname{Re} \int \mathcal{D}\left[\phi, \phi^{\dagger}, H\right] x_{i}^{*} x_{i} \mathrm{e}^{-S_{H}}
$$

Here $\phi=\left(x_{i}, \psi_{i}\right)^{T}, \phi^{\dagger}=\left(x_{i}^{*}, \bar{\psi}_{i}\right)$ is a vector with $N$ bosonic and $N$ fermionic components. We set the variance of $H$ to $g=1 / 2$. The action reads

$$
S_{H}=\mathrm{i} \phi_{i}^{\dagger}\left(H_{i j}-z \delta_{i j}\right) \phi_{j}+\operatorname{tr} H^{2}
$$

Integration over $H$ yields an effective action

$$
S_{4}=-\mathrm{i} z \phi_{i}^{\dagger} \phi_{i}+\frac{1}{4} \operatorname{trg}\left(\phi_{i} \phi_{i}^{\dagger}\right)^{2}
$$

A Hubbard-Stratonovich transformation to $\mathbf{Q}$-variables (the $q, p$ are bosonic and the $\vartheta, \bar{\vartheta}$ are fermionic),

$$
\mathbf{Q}=\left(\begin{array}{cc}
q & \bar{\vartheta} \\
\vartheta & \mathrm{i} p
\end{array}\right)
$$

gives an action bilinear in $\phi$ :

$$
S_{\mathbf{Q}}=\mathrm{i} \phi_{i}^{\dagger}(\mathbf{Q}-z) \phi_{i}+\operatorname{trg} \mathbf{Q}^{2}
$$

Now we integrate over the $\phi$-fields and the Grassmannvariables $\vartheta, \bar{\vartheta}$ exactly to get an action, which depends only on the two real variables $q$ and $p$. The DOS reads

$$
\langle n(z)\rangle=\frac{-2}{\pi N} \operatorname{Im} \int \mathcal{D}[\mathbf{Q}] \frac{q \mathrm{e}^{-\operatorname{trg} \mathbf{Q}^{2}}}{\operatorname{detg}(\mathbf{Q}-z)^{N}}=\frac{-2}{\pi N} \operatorname{Im} \int \frac{\mathrm{d} p \mathrm{~d} q}{\pi} q \mathrm{e}^{-p^{2}-q^{2}} \frac{(\mathrm{i} p-z)^{N}}{(q-z)^{N}}\left(1-\frac{N}{2(q-z)(\mathrm{i} p-z)}\right)
$$

If we now set $z=E+\mathrm{i} \varepsilon$ with $E \in \mathbb{R}$ and perform the integration over $p$ and $q$, we get the exact finite $-N$ result 3

$$
\langle n(E)\rangle=\frac{\mathrm{e}^{-E^{2}}}{2^{N} N ! \sqrt{\pi}}\left(H_{N}^{2}(E)-H_{N+1}(E) H_{N-1}(E)\right)
$$

duce a rescaled energy variable $x=E / \sqrt{N}$. With this scaling the band width becomes finite and the DOS a continuous normalizable distribution in the limit $N \rightarrow \infty$. After the rescaling (which is done for $p$ and $q$ as well) we get for the DOS (after integration over $\vartheta, \frac{\vartheta}{\vartheta}$ but before integration over $p$ and $q$ )

where $H_{n}(E)$ denotes the $n$-th Hermite polynomial. To perform an expansion around the limit $N=\infty$, we intro-

$$
\langle n(x)\rangle=\frac{-2}{\pi} \operatorname{Im} \int \frac{\mathrm{d} p \mathrm{~d} q}{\pi / N} q \mathrm{e}^{-N p^{2}-N q^{2}} \frac{(\mathrm{i} p-x)^{N}}{(q-x)^{N}}\left(1-\frac{1}{2(q-x)(\mathrm{i} p-x)}\right),
$$

which can be written as

$$
\langle n(x)\rangle=\int \mathrm{d} p \mathrm{~d} q \mathrm{e}^{-N S(p, q, x)} f(p, q, x) .
$$

The large prefactor $N$ in the exponent allows for a saddlepoint approximation of the $p, q$-integrals in the usual 
way. We get four solutions of the saddle-point equations

$$
q_{ \pm}=x / 2 \pm \mathrm{i} / 2 \sqrt{2-x^{2}}, \quad p_{ \pm}=-\mathrm{i} q_{ \pm} .
$$

The stability matrix is

$$
\begin{aligned}
\frac{\partial^{2} S}{2 \partial q^{2}} & =1-q_{ \pm}^{2}= \pm \mathrm{i} \sqrt{2-x^{2}} q_{ \pm}=: \Delta_{ \pm} \\
\frac{\partial^{2} S}{2 \partial p^{2}} & =\Delta_{ \pm} \\
\frac{\partial^{2} S}{\partial p \partial q} & =0 .
\end{aligned}
$$

Figures 1 and 2 show the location of the saddle-points in the complex plane along with their stable directions and possible integration paths. The integral (6) converges

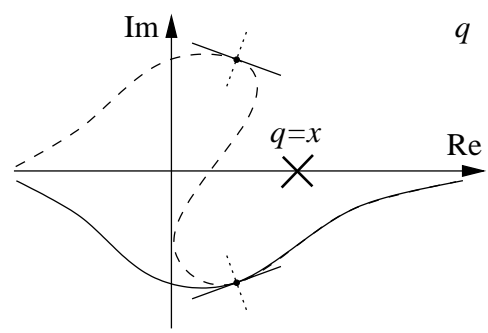

FIG. 1: The saddle-points of $q$ and possible integration paths. Solid lines denote the stable directions and dotted lines the unstable directions.

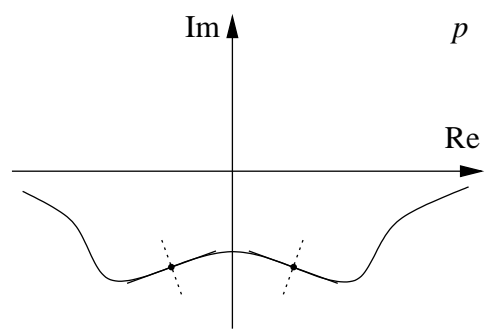

FIG. 2: The saddle-points for $p$.

only for $\operatorname{Im} z>0$. This means, that the integrationpath of $q$ must pass below the singularity at $q=x$. This forces us to use only the saddle-point $q_{-}$of the $q$-integration: A path of integration which also crosses $q_{+}$leads through the region between the two saddlepoints, where the integrand of (8) is large. Therefore the correct choice for the path of integration over $q$ is the solid line in figure 1. In contrast it is possible to use an integration-path which goes through both saddle-points for the $p$-integral, which is shown in figure 2.

After the change of variables

$$
q \rightarrow q_{-}+\frac{\delta q}{\sqrt{N \Delta_{-}}} \quad p \rightarrow p_{ \pm}+\frac{\delta p}{\sqrt{N \Delta_{ \pm}}}
$$

we may perform an expansion in $\delta q$ and $\delta p$, which defines the $1 / N$-expansion. The action $S_{S P}^{(1)}=S\left(q_{-}, p_{-}\right)$vanishes and yields the well-known semi-circle law as the only contribution surviving in the $N \rightarrow \infty$-limit. The action $S_{S P}^{(2)}=S\left(q_{-}, p_{+}\right)$does not vanish but is purely imaginary and yields a contribution $\sim e^{-N S_{S P}^{(2)}}$ of modulus 1 . This contribution disappears in the $N=\infty$-limit, because the factor $\left(1-\frac{1}{2(q-x)(\mathrm{i} p-x)}\right)$ vanishes for $q=q_{-}, p=p_{+}$. It yields, however, an important oscillatory contribution $\propto 1 / N$, whereas the (non-oscillatory) corrections to the first saddle-point $\left(q_{-}, p_{-}\right)$start with order $1 / N^{2}$. The final result is

$$
\langle n(x)\rangle=\frac{\sqrt{2-x^{2}}}{\pi}-\frac{(-1)^{N} \cos \left[N S_{0}(x)\right]}{\pi \sqrt{2} N\left(2-x^{2}\right)}+\mathcal{O}\left(\frac{1}{N^{2}}\right)
$$

with

$$
S_{0}(x)=S_{S P}^{(2)}(x)=x \sqrt{2-x^{2}}+2 \arcsin (x / \sqrt{2})
$$

and

$$
S_{0}^{\prime}(x)=2 \sqrt{2-x^{2}}=2 \pi n_{0}(x) .
$$

where the zeroth order approximation to the DOS (the semi-circle) is written as $n_{0}(x)=(1 / \pi) \sqrt{2-x^{2}}$. The exact density of states and the $1 / N-$ approximation is shown in figure 3. From (16) and (17) we note that the

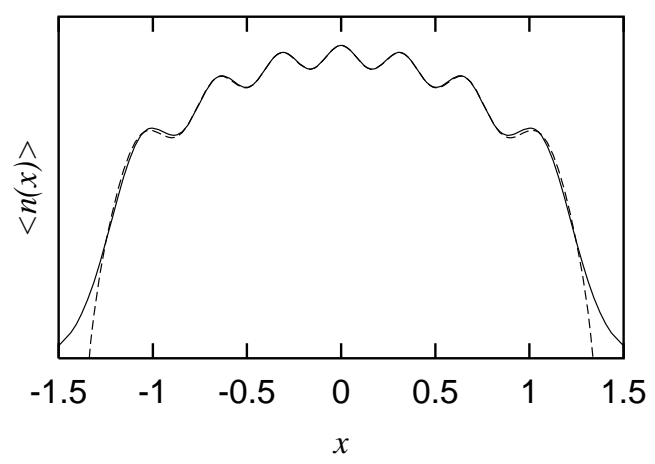

FIG. 3: Exact DOS of the GUE for $N=7$. The dashed line is the $1 / N$-approximation.

number of maxima of the oscillatory $1 / N$-term equals $N$ and the local width between two maxima scales as the inverse of the DOS in zeroth approximation. The positions of the maxima give therefore the locations of eigenvalues for a "typical" realization of a random matrix from the GUE with one state per maximum on average. Identifying the distance between to adjacent maxima with the average level spacing at $x=E / \sqrt{N}$, we get for large $N$ the following expression for $\Delta(E)$ :

$$
\Delta(E) \approx \frac{1}{\sqrt{N} n_{0}(E / \sqrt{N})}
$$

which is valid for $E$ not too close to the band edge. The $1 / N$-expansion diverges obviously at the band edge, because the saddle-points coalesce and as a consequence the stability matrix vanishes. 


\section{THE OTHER ENSEMBLES}

The elements of the orthogonal, symplectic and Class $\mathrm{C} / \mathrm{D}$ ensembles are conveniently represented as $2 N \times 2 N$ matrices having a $2 \times 2$-block structure:

$$
H=\left(\begin{array}{ll}
A & B \\
C & D
\end{array}\right),
$$

where the $N \times N$-matrices $A, B, C, D$ fulfill a set of conditions defining the different ensembles. We set the variance of $H$ to $g=1$ in the following, which yields a bandwidth of 4 in all cases.

\section{The orthogonal Ensemble}

The elements of the GOE are real symmetric $2 N \times 2 N$ matrices: $H=H^{T}=H^{*}$. To simplify calculations, it is convenient to use instead of $H$ the unitary equivalent ma$\operatorname{trix} M=U H U^{\dagger}$ with $U=\exp \left(\mathrm{i}(\pi / 4) \sigma_{1}\right) \otimes \mathbb{1}_{N}$, where $\sigma_{1}$ is a Pauli matrix acting on the $2 \times 2$-blocks. If $H$ is real symmetric, $M$ fulfills the relation $M=\sigma_{1} M^{T} \sigma_{1}$. This entails the following relation among the block matrices

$$
M=\left(\begin{array}{cc}
A & B \\
B^{\dagger} & A^{T}
\end{array}\right),
$$

where $A$ is a hermitian $N \times N$-matrix and $B$ is complex symmetric. As in section II, we write for the DOS

$$
\langle n(z)\rangle=\frac{1}{2 \pi N} \operatorname{Re} \int \mathcal{D}\left[\phi, \phi^{\dagger}, M\right]\left(x_{1 i}^{*} x_{1 i}\right) \mathrm{e}^{-S},
$$

with $\phi=\left(x_{1}, x_{2}, \psi_{1}, \psi_{2}\right)^{T}$, where the $x_{1 / 2}, \psi_{1 / 2}$ are $N$-dimensional complex bosonic, resp. fermionic vectors and $S$ reads

$$
S=\mathrm{i} \phi^{\dagger}(M-z) \phi+\frac{1}{2} \operatorname{tr} M^{2} .
$$

Now we introduce the notation

$$
\chi_{1 j}=\left(x_{1 j}, x_{2 j}^{*}, \psi_{1 j}, \bar{\psi}_{2 j}\right)^{T}, \quad \chi_{2 j}=\left(x_{2 j}, x_{1 j}^{*}, \psi_{2 j}, \bar{\psi}_{1 j}\right)^{T}
$$

( $j$ is the index of the $N$-dimensional tensor component) together with the transposition

$$
\chi_{1 j}^{t}=\left(x_{1 j}^{*}, x_{2 j}, \psi_{1 j}^{*},-\psi_{2 j}\right), \chi_{2 j}^{t}=\left(x_{2 j}^{*}, x_{1 j}, \psi_{2 j}^{*},-\psi_{1 j}\right) .
$$

After Integration over $M$, we get the quartic action in $\chi, \chi^{t}$ :

$$
S_{4}=-\mathrm{i} \chi_{1 i}^{t} \chi_{1 i}+\frac{1}{8} \operatorname{trg}\left(\chi_{1 i} \chi_{1 i}^{t}+\chi_{2 i} \chi_{2 i}^{t}\right)^{2} .
$$

After transformation to the Q-matrix

$$
\mathbf{Q}=\left(\begin{array}{cccc}
q_{1} & q_{2}^{*} & \bar{\vartheta}_{1} & -\vartheta_{2} \\
q_{2} & q_{1} & \bar{\vartheta}_{2} & -\vartheta_{1} \\
\vartheta_{1} & \vartheta_{2} & \mathrm{i} p & 0 \\
\bar{\vartheta}_{2} & \bar{\vartheta}_{1} & 0 & \mathrm{i} p
\end{array}\right)
$$

we obtain

$$
\langle n(z)\rangle=\frac{-1}{\pi N} \operatorname{Im} \int \mathcal{D}[\mathbf{Q}] \frac{q_{1} \mathrm{e}^{-\frac{1}{2} \operatorname{trg} \mathbf{Q}^{2}}}{\operatorname{detg}(\mathbf{Q}-z)^{N}},
$$

with

$$
\begin{aligned}
& \operatorname{detg} \mathbf{Q}= \\
& \frac{Q}{(\mathrm{i} p)^{2}} \exp \left(-\frac{2}{Q(\mathrm{i} p)}\left(q_{1} \Theta_{1}-q_{2} \Theta_{2}-q_{2}^{*} \bar{\Theta}_{2}\right)-\frac{2 \Theta_{2} \bar{\Theta}_{2}}{Q(\mathrm{i} p)^{2}}\right)
\end{aligned}
$$

and $Q=q_{1}^{2}-q_{2} q_{2}^{*}, \Theta_{1}=\bar{\vartheta}_{1} \vartheta_{1}+\bar{\vartheta}_{2} \vartheta_{2}, \Theta_{2}=\bar{\vartheta}_{1} \vartheta_{2}$. Note that the Grassmann-integration contains now quartic terms in $\vartheta, \bar{\vartheta}$, which can nevertheless be done exactly. The result is $\left(q=q_{1}, r=q_{2} q_{2}^{*}\right)$ :

$$
\langle n(z)\rangle=\frac{-1}{\pi N} \operatorname{Im} \int \frac{\mathrm{d} p \mathrm{~d} q}{\pi} \mathrm{d} r \mathrm{e}^{-p^{2}-q^{2}-r} q \frac{(\mathrm{i} p-E)^{2 N}}{\left[(q-z)^{2}-r\right]^{N}}\left(1-\frac{2 N(q-z)}{\left[(q-z)^{2}-r\right](\mathrm{i} p-z)}+\frac{(2 N-1) N}{2\left[(q-z)^{2}-r\right](\mathrm{i} p-z)^{2}}\right) .
$$

Setting now as above $z=E+\mathrm{i} \varepsilon$ and integrating over $p, q$ and $r$, we obtain the exact DOS in terms of Hermitepolynomials and the error-function:

$$
\langle n(E)\rangle=\frac{\mathrm{e}^{-E^{2}}}{4^{N} N \sqrt{\pi}}\left(\frac{H_{2 N-1}(E)^{2}-H_{2 N}(E) H_{2 N-2}(E)}{(2 N-2) !}-\frac{H_{2 N-1}(E)}{(N-1) !}\left(\sum_{k=1}^{N-1} \frac{2 k !}{(2 k) !} H_{2 k-1}(E)-\sqrt{\frac{\pi}{2}} \mathrm{e}^{\frac{E^{2}}{2}} \mathrm{erf} \frac{E}{\sqrt{2}}\right)\right)
$$

\section{The symplectic Ensemble}

Here we have

$$
H=\left(\begin{array}{cc}
A & B \\
B^{\dagger} & A^{T}
\end{array}\right)
$$

with $A$ hermitian and $B$ complex antisymmetric. Proceeding as in the case of the GOE (the definition of $\chi, \chi^{t}$ 
is the same), we find the quartic action

$$
S_{4}=\frac{1}{8} \operatorname{trg}\left(\chi_{1 i} \chi_{1 i}^{t}+\Sigma_{3} \chi_{2 i} \chi_{2 i}^{t} \Sigma_{3}\right)^{2},
$$

where $\Sigma_{3}=\sigma_{3} \otimes \mathbb{1}_{N}$. The corresponding $\mathbf{Q}$-matrix reads now

$$
\mathbf{Q}=\left(\begin{array}{cccc}
q & 0 & \bar{\vartheta}_{1} & \bar{\vartheta}_{2} \\
0 & q & -\vartheta_{2} & -\vartheta_{1} \\
\vartheta_{1} & \bar{\vartheta}_{2} & \mathrm{i} p_{1} & \mathrm{i} p_{2}^{*} \\
\vartheta_{2} & \bar{\vartheta}_{1} & \mathrm{i} p_{2} & \mathrm{i} p_{1}
\end{array}\right)
$$

and the DOS reads,

$$
\langle n(z)\rangle=\frac{-1}{\pi N} \operatorname{Im} \int \mathcal{D}[\mathbf{Q}] \frac{q \mathrm{e}^{-\frac{1}{2} \operatorname{trg} \mathbf{Q}^{2}}}{\operatorname{detg}(\mathbf{Q}-z)^{N}}
$$

with

$$
\begin{aligned}
& \operatorname{detg} \mathbf{Q}^{-1}= \\
& \frac{P}{q^{2}} \exp \left(\frac{2}{P q}\left(\mathrm{i} p_{1} \Theta_{1}-\mathrm{i} p_{2} \Theta_{2}-\mathrm{i} p_{2}^{*} \bar{\Theta}_{2}\right)-\frac{2 \Theta_{2} \bar{\Theta}_{2}}{P q^{2}}\right)
\end{aligned}
$$

and $P=\left(\mathrm{i} p_{1}\right)^{2}-\left(\mathrm{i} p_{2}\right)\left(\mathrm{i} p_{2}^{*}\right), \Theta_{1}=\bar{\vartheta}_{1} \vartheta_{1}+\bar{\vartheta}_{2} \vartheta_{2}$ and $\Theta_{2}=$ $\bar{\vartheta}_{2} \vartheta_{1}$. Setting $p=p_{1}$ and $r=p_{2} p_{2}^{*}$, eq.(34) reads after integration over the Grassmann variables,

$$
\langle n(z)\rangle=\frac{-1}{\pi N} \operatorname{Im} \int \frac{\mathrm{d} p \mathrm{~d} q}{\pi} \mathrm{d} r q \mathrm{e}^{-p^{2}-q^{2}-r} \frac{\left[(\mathrm{i} p-z)^{2}+r\right]^{N}}{(q-z)^{2 N}}\left(1-\frac{2 N(\mathrm{i} p-z)}{\left[(\mathrm{i} p-z)^{2}+r\right](q-z)}+\frac{(2 N+1) N}{2\left[(\mathrm{i} p-z)^{2}+r\right](q-z)^{2}}\right)
$$

and

$$
\langle n(E)\rangle=\frac{\mathrm{e}^{-E^{2}}}{(2 N) ! \sqrt{\pi}}\left(\frac{1}{4^{N}}\left(H_{2 N}(E)^{2}-H_{2 N+1}(E) H_{2 N-1}(E)\right)-\frac{N !}{2 N} H_{2 N}(E) \sum_{k=0}^{N-1} \frac{H_{2 k}(E)}{4^{k} k !}\right) .
$$

\section{Class D}

The elements of class D are parameterized as

$$
H=\left(\begin{array}{cc}
A & B \\
B^{\dagger} & -A^{T}
\end{array}\right)
$$

with $A$ hermitian and $B$ complex antisymmetric. The quartic action reads (in the notation above)

$$
S_{4}=\frac{1}{8} \operatorname{trg}\left(\left(\chi_{1 i} \chi_{1 i}^{t}+\chi_{2 i} \chi_{2 i}^{t}\right) \Sigma_{3}\right)^{2},
$$

and the Q-matrix

$$
\mathbf{Q}=\left(\begin{array}{cccc}
q_{1} & q_{2}^{*} & -\vartheta_{1} & -\vartheta_{2} \\
q_{2} & -q_{1} & \bar{\vartheta}_{2} & \bar{\vartheta}_{1} \\
\bar{\vartheta}_{1} & \vartheta_{2} & \mathrm{i} p & 0 \\
\bar{\vartheta}_{2} & \vartheta_{1} & 0 & -\mathrm{i} p
\end{array}\right)
$$

For $\langle n(z)\rangle$ we get

$$
\langle n(z)\rangle=\frac{-1}{\pi N} \operatorname{Im} \int \mathcal{D}[\mathbf{Q}] \frac{q_{1} \mathrm{e}^{-\frac{1}{2} \operatorname{trg} \mathbf{Q}^{2}}}{\operatorname{detg}\left(\Sigma_{3} \mathbf{Q}-z\right)^{N}},
$$

with

$$
\begin{aligned}
& \operatorname{detg} \Sigma_{3} \mathbf{Q}= \\
& \frac{Q}{(\mathrm{i} p)^{2}} \exp \left(-\frac{2}{Q(\mathrm{i} p)}\left(q_{1} \Theta_{1}-q_{2} \Theta_{2}+q_{2}^{*} \bar{\Theta}_{2}\right)-\frac{2 \Theta_{2} \bar{\Theta}_{2}}{Q(\mathrm{i} p)^{2}}\right)
\end{aligned}
$$

and $Q=q_{1}^{2}+q_{2} q_{2}^{*}, \Theta_{1}=\bar{\vartheta}_{1} \vartheta_{1}-\bar{\vartheta}_{2} \vartheta_{2}, \Theta_{2}=\vartheta_{1} \vartheta_{2}$. The integrand of (41) contains a pole depending on $q=q_{1}$ and $r=q_{2} q_{2}^{*}$. To circumvent it, we have to deform the integration path into the complex plane:

$$
\begin{aligned}
q & \rightarrow \mathrm{e}^{-\mathrm{i} \pi / 4} q+\varepsilon \mathrm{e}^{\mathrm{i} \pi / 4} \tanh (q / \varepsilon) \\
\sqrt{r} & \rightarrow \mathrm{e}^{\mathrm{i} \pi / 4} \sqrt{r}+\varepsilon \mathrm{e}^{-\mathrm{i} \pi / 4} \tanh (\sqrt{r} / \varepsilon)
\end{aligned}
$$

for $\operatorname{Re} z>0$ and $\varepsilon$ must be small enough: $0<\varepsilon<$ $(\operatorname{Im} z+\operatorname{Re} z) / \sqrt{2}$. We find then 


$$
\langle n(z)\rangle=\frac{-1}{\pi N} \operatorname{Im} \int \frac{\mathrm{d} p \mathrm{~d} q}{\pi} \mathrm{d} r q \mathrm{e}^{-p^{2}-q^{2}-r} \frac{(\mathrm{i} p-z)^{2 N}}{\left[(q-z)^{2}+r\right]^{N}}\left(1-\frac{N(2 N-1)}{2\left[(q-z)^{2}+r\right](\mathrm{i} p-z)^{2}}\right)
$$

and

$$
\langle n(E)\rangle=\frac{-(-1)^{N} \mathrm{e}^{-E^{2}}}{N ! 4^{N} \sqrt{\pi}}\left(\frac{(-1)^{N}(N-1) !}{(2 N-2) !} H_{2 N}(E) H_{2 N-2}(E)+2 E H_{2 N-1}(E)\left(\sum_{k=1}^{N-1} \frac{(-1)^{k} k !}{(2 k) !} H_{2 k}(E)+1+\frac{1}{2 E^{2}}\right)\right)
$$

\section{Class C}

The parameterization reads

$$
H=\left(\begin{array}{cc}
A & B \\
B^{\dagger} & -A^{T}
\end{array}\right)
$$

with $A$ hermitian and $B$ complex symmetric. The quartic action is:

$$
S_{4}=\operatorname{trg}\left(\chi_{1 i} \chi_{1 i}^{t} \Sigma_{3}+\Sigma_{3} \chi_{2 i} \chi_{2 i}^{t}\right)^{2}
$$

with the $\mathbf{Q}$-matrix

$$
\left(\begin{array}{cccc}
q & 0 & -\vartheta_{1} & \bar{\vartheta}_{2} \\
0 & -q & -\vartheta_{2} & \bar{\vartheta}_{1} \\
\bar{\vartheta}_{1} & \bar{\vartheta}_{2} & \mathrm{i} p_{1} & \mathrm{i} p_{2}^{*} \\
\vartheta_{2} & \vartheta_{1} & \mathrm{i} p_{2} & -\mathrm{i} p_{1}
\end{array}\right)
$$

We have

$$
\langle n(z)\rangle=\frac{-1}{\pi N} \operatorname{Im} \int \mathcal{D}[\mathbf{Q}] \frac{q_{1} \mathrm{e}^{-\frac{1}{2} \operatorname{trg} \mathbf{Q}^{2}}}{\operatorname{detg}\left(\Sigma_{3} \mathbf{Q}-z\right)^{N}}
$$

with

$$
\begin{aligned}
& \operatorname{detg}\left(\Sigma_{3} \mathbf{Q}\right)^{-1}= \\
& \frac{P}{q^{2}} \exp \left(\frac{2}{P q}\left(\mathrm{i} p_{1} \Theta_{1}+\mathrm{i} p_{2} \Theta_{2}-\mathrm{i} p_{2}^{*} \bar{\Theta}_{2}\right)-\frac{2 \Theta_{2} \bar{\Theta}_{2}}{P q^{2}}\right)
\end{aligned}
$$

and $P=\left(\mathrm{i} p_{1}\right)^{2}+\left(\mathrm{i} p_{2}\right)\left(\mathrm{i} p_{2}^{*}\right), \Theta_{1}=\bar{\vartheta}_{1} \vartheta_{1}-\bar{\vartheta}_{2} \vartheta_{2}$ and $\Theta_{2}=$ $\bar{\vartheta}_{1} \bar{\vartheta}_{2}$. With $p=p_{1}, r=p_{2} p_{2}^{*}$ the result reads

$$
\begin{aligned}
\langle n(z)\rangle & =\frac{-1}{\pi N} \operatorname{Im} \int \frac{\mathrm{d} p \mathrm{~d} q}{\pi} \mathrm{d} r \mathrm{e}^{p^{2}-q^{2}-r} q \frac{\left[(\mathrm{i} p-z)^{2}-r\right]^{N}}{(q-z)^{2 N}}\left(1-\frac{N(2 N+1)}{2\left[(\mathrm{i} p-z)^{2}-r\right](q-z)^{2}}\right) \\
\langle n(E)\rangle & =\frac{(-1)^{N} N ! \mathrm{e}^{-E^{2}}}{(2 N) ! \sqrt{\pi}}\left(H_{2 N}(E) \sum_{k=0}^{N} \frac{(-1)^{k} H_{2 k}(E)}{k ! 4^{k}}+\frac{H_{2 N+2}(E)}{4 N} \sum_{k=0}^{N-1} \frac{(-1)^{k} H_{2 k}(E)}{k ! 4^{k}}\right) .
\end{aligned}
$$

\section{SADDLE-POINT APPROXIMATION}

The saddle-point approximation for the four ensembles proceeds as in the unitary case in general: All four ensembles share the saddle-points $q_{ \pm}=x / 2 \pm \mathrm{i} \sqrt{1-x^{2} / 4}$, $p_{ \pm}=-\mathrm{i} q_{ \pm}, r_{0}=0,(x=E / \sqrt{N})$. As in the unitary case, the points $\left(q_{+}, p_{ \pm}, r_{0}\right)$ have to be avoided by the integration path. The fluctuations are quadratic in $p$ and $q$ and linear in $r$ around the two remaining SP's $\left(q_{-}, p_{ \pm}, r_{0}\right)$. Apart from them, we have additional SP's for the GOE and the GSE, given in Table $[$.

The additional SP can not be reached in the GOE case $\left(r_{S P}<0\right)$ but has to be included for the GSE $\left(r_{S P}>0\right)$. In class $\mathrm{C}$ and $\mathrm{D}$ there is an additional saddle-point man- ifold at $x=0$. However, our results displayed below are obtained by confining the analysis to the standard saddlepoints, which exist for all values of $x$, thereby assuming continuity in the limit $x \rightarrow 0$. In principle this procedure could have led to an additional singularity at $x=0$ similar to the divergence at the band edge. But the comparison with the exact DOS in these cases shows that our assumption is indeed correct and the additional saddlepoints play no role in computing the $1 / N$-expansion.

We give in the following the results for the $1 / N$ expansion of the GOE, GSE and Class C/D. 
TABLE I: The additional saddle-points for the GOE and the GSE

\begin{tabular}{lccc}
\hline \hline Ensemble & $p$ & $q$ & $r$ \\
GOE & $-\mathrm{i}\left(\frac{x}{2} \pm \mathrm{i} \sqrt{1-\frac{x^{2}}{4}}\right)$ & $\frac{x}{2}$ & $\frac{x^{2}}{4}-1$ \\
GSE & $-\mathrm{i} \frac{x}{2}$ & $\frac{x}{2} \pm \mathrm{i} \sqrt{1-\frac{x^{2}}{4}}$ & $1-\frac{x^{2}}{4}$ \\
\hline \hline
\end{tabular}

\section{The orthogonal Ensemble}

Lets define the $N=\infty$ approximation to the DOS of the GOE as $n_{0}(x)=(1 / \pi) \sqrt{1-x^{2} / 4}$. Then the $1 / N$-expansion reads to order $1 / N^{2}$

$$
\langle n(x)\rangle=n_{0}(x)-\frac{1}{N} \frac{1}{8 \pi^{2} n_{0}(x)}+\frac{1}{N^{2}} \frac{3+x^{2}}{128 \pi^{6} n_{0}(x)^{5}}-\frac{1}{N^{2}} \frac{1}{64 \pi^{6} n_{0}(x)^{5}} \cos \left[2 N S_{0}(x)-\arcsin (x / 2)\right]
$$

with

$$
\begin{aligned}
& S_{0}(x)=2 \arcsin (x / 2)+x \sqrt{1-x^{2} / 4} \\
& S_{0}^{\prime}(x)=2 \pi n_{0}(x)
\end{aligned}
$$

analogous to the unitary case. We observe the same features as in the GUE case: The number of maxima of the oscillatory part within the band $-2<x<2$ given by the semi-circle is $2 N$, the total number of levels, so we have one level per maximum. The correction diverges close to the band edge as expected. Apart from the oscillatory contribution there is a non-oscillatory contribution of or$\operatorname{der} 1 / N$. However, the information about the level repulsion is encoded in the oscillating part $\propto \cos \left(2 N S_{0}(x)\right)$. This term is of order $1 / N^{2}$, which means that the repulsion of levels is weaker in the GOE than in the GUE. Formally we can write

$$
\begin{aligned}
\langle n(x)\rangle= & \left.n_{0}(x)+\frac{1}{N} \text { (non-oscillating term }\right) \\
& \left.+\frac{1}{N^{\alpha}} \text { (oscillating term }\right)
\end{aligned}
$$

with $\alpha=2$. For the GUE $\alpha=1$. Now the two-level correlation fupction $R_{2}(r)$ behaves for the Wigner-Dyson Ensembles as 3 :

$$
R_{2}(r) \sim r^{\beta} \quad \text { for } \quad r \ll 1,
$$

where $r$ is the distance between two levels on a scale corresponding to the average level spacing $\Delta(x) \sim 1$. For the GUE we have $\beta=2$ and for the $\operatorname{GOE} \beta=1$. We conjecture therefore

$$
\beta=\frac{2}{\alpha}
$$

as a relation between the universal parameter $\beta$, which characterizes the short distance behavior of the twolevel correlation function and the exponent $\alpha$ of the factor $1 / N$, which multiplies the oscillating term in the $1 / N$-expansion. With this relation we can extract from the $1 / N$-expansion of the one-point function universal information about the two-point function, which discerns the WD-Ensembles from each other (whereas the DOS is the same for all three ensembles in the limit $N=\infty$ ).

\section{The symplectic Ensemble}

We have (with the same definition of $n_{0}(x)$ and $S_{0}(x)$ as in the GOE)

$$
\begin{aligned}
\langle n(x)\rangle= & n_{0}(x)+\frac{1}{N} \frac{1}{8 \pi^{2} n_{0}(x)} \\
& -\frac{1}{N^{\frac{1}{2}}} \frac{\cos \left[N S_{0}(x)+\frac{1}{2} \arcsin (x / 2)\right]}{(-1)^{N} \sqrt{8} \pi^{\frac{3}{4}} n_{0}(x)^{\frac{1}{4}}} .
\end{aligned}
$$

Because each eigenvalue appears twice in a $2 N \times 2 N$ matrix from the symplectic ensemble due to Kramers degeneracy 3 , we have only $N$ different eigenvalues and therefore $N$ maxima within the band. The oscillatory term comes with a $1 / \sqrt{N}$ prefactor, therefore $\alpha=1 / 2$, which gives with (59) the correct universal exponent $\beta=4$.

\section{Class C}

The $1 / N$-expansion yields

$$
\begin{aligned}
\langle n(x)\rangle= & n_{0}(x)+\frac{1}{N} \frac{1}{8 \pi^{2} n_{0}(x)} \\
& -\frac{1}{N} \frac{\sin \left[2 N S_{0}(x)+\arcsin (x / 2)\right]}{4 \pi^{3} x n_{0}(x)^{2}} .
\end{aligned}
$$

Each element of the Class $\mathrm{C}$ ensemble has a spectrum symmetric with respect to $x=0$ : To each eigenvalue $x$ there is a state with eigenvalue $-x$. No matrix from Class $\mathrm{C}$ has an eigenvalue zero. Therefore the DOS vanishes at $x=0$. The number of maxima in the band equals $2 N$, we have again one (non-degenerate) level per maximum. Now the spectral region around $x=0$ is different from the WD-Ensembles because there is no smooth limit of $\langle n(x)\rangle$ for $N \rightarrow \infty$ : If one introduces the rescaled variable $y=2 N x / \pi$ and considers the microscopic limit $x \rightarrow 0$, $N \rightarrow \infty$, keeping $y$ finite, the DOS reads

$$
\langle n(y)\rangle=\frac{1}{\pi}\left(1-\frac{\sin (2 \pi y)}{2 \pi y}\right)
$$



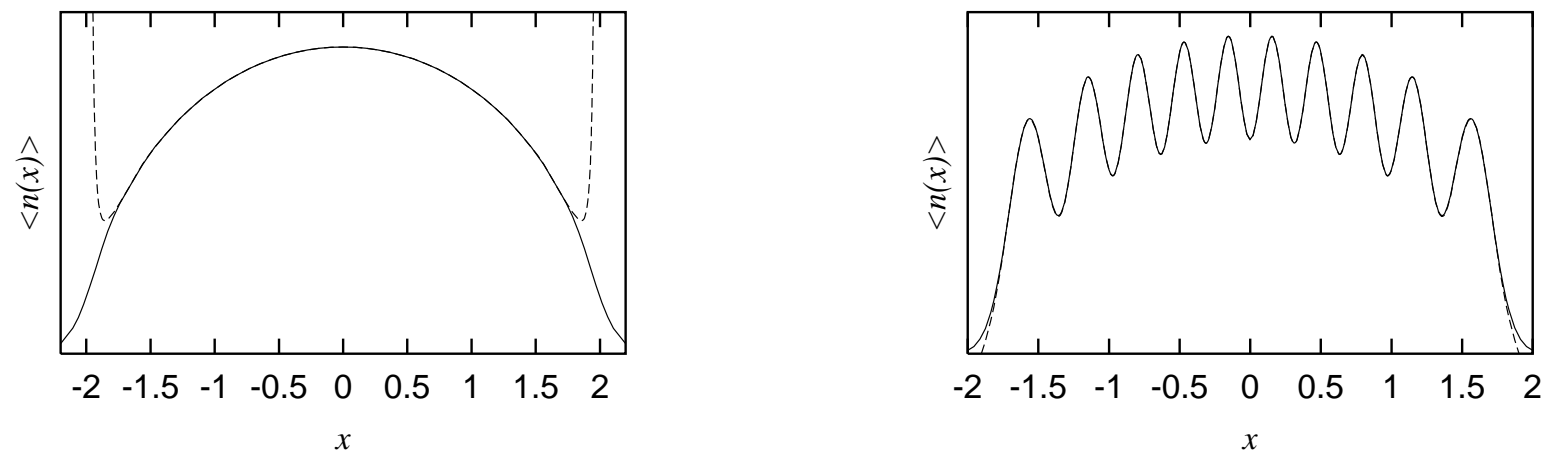

FIG. 4: The GOE and the GSE. $N=10$ in both cases. The solid line is the exact DOS, the dashed line is the saddle-point approximation.
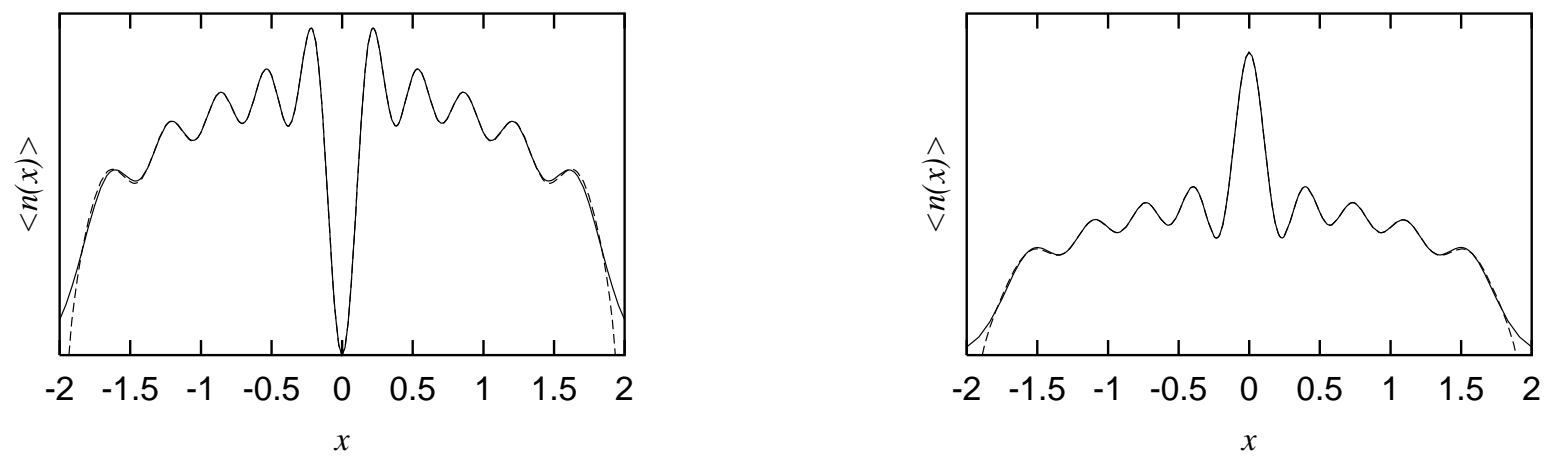

FIG. 5: Class C and Class D. $N=5$ in both cases.The solid line is the exact DOS, the dashed line is the saddle-point approximation.

which coincides with the result in [2]. In terms of $y$, the correction term is independent of $N$ in contrast to the GUE, where the oscillatory contribution to the DOS vanishes as $1 / N$ for large $N$. This, however, is not a signal of an enhanced level repulsion close to $x=0$ in Class C. It is merely due to the lack of (approximate) translational invariance in the band center, which is caused by the mirror-symmetry mentioned above. In the GUE, translational invariance is broken only through the term proportional to $\exp \left(-\sum_{i=1}^{N} \lambda_{i}^{2}\right)$ in the joint probability distribution of the eigenvalues $\lambda_{i}$, which can be neglected close to the band center and large $N$. Therefore the oscillatory structure in $\langle n(x)\rangle$ is smeared out by the summation over all members of the ensemble, whereas in Class $\mathrm{C}$ the quotient of the probability to find a level at the first maximum respective the first minimum of $\langle n\rangle$ is independent of $N$. The spectrum is therefore more rigid near $x=0$. The two-level correlation function $R_{2}\left(y_{1}, y_{1}+y\right)$ behaves for $0<y_{1}, y \ll 1$ nevertheless as $y^{2}$ and belongs therefore to the unitary universality class 2 . The correction term does not modify the macroscopic behavior of $\langle n\rangle$ because the function $\sin (N x) /(N x)$ tends to zero in the $L_{2}-$ sense for $N \rightarrow \infty$. That means that the probability to find $m$ states in a region of width $\Delta x=\frac{\pi m}{2 N}$ around zero tends to one for $1 \ll m \ll N$ in the limit
$N \rightarrow \infty$, as in the WD-Ensembles.

Because the $1 / N$-approximation is reliable everywhere except at the band edge, we can compute the average level spacing exactly in the vicinity of $x=0$ without recourse to measure $x$ (respective $y$ ) in units of the mean spacing at a distance of many spacings from zerot. In terms of $y$ (which is exactly related to $x$ and the original variable $E$ through $N$ ), the average spacing between the first and second level $>0$ is given by

$$
\left\langle\Delta_{y}(1,2)\right\rangle=\frac{1}{2 \pi}\left(z_{4}-z_{2}\right) \approx 1,
$$

where $z_{k}>0$ denotes the $k$-th zero of $\tan (z)-z$. This relation is valid for large $N$ but $\Delta(j, j+1)$ can be computed via (61) for all levels $j$ and $j+1$ not too close to the band edge and arbitrary $N$. 


\section{Class D}

The same mirror-symmetry as in Class $\mathrm{C}$ is valid in Class D. We have

$$
\begin{aligned}
\langle n(x)\rangle= & n_{0}(x)-\frac{1}{N} \frac{1}{8 \pi^{2} n_{0}(x)} \\
& +\frac{1}{N} \frac{\sin \left[2 N S_{0}(x)-\arcsin (x / 2)\right]}{4 \pi^{3} x n_{0}(x)^{2}}
\end{aligned}
$$

very similar to Class $\mathrm{C}$, but now the DOS is enhanced in the band center because there are always two states with eigenvalue close to zero. The number of different maxima is $2 N-1$. Again this feature vanishes in the $N=\infty$-limit, because the single additional state at $x=0$ has measure zero for vanishing average level distance $\Delta(E) \sim 1 / N$. As in the Class C ensemble we have $\alpha=1$ and the universal exponent is $\beta=2$. Class $\mathrm{D}$ belongs therefore with Class $\mathrm{C}$ to the unitary universality class, which determines the level repulsion even in the immediate vicinity of $E=0$ e.g. for the second and third state away from zero.

Figures 4 and 5 give the exact DOS and the $1 / N$ approximation (dashed lines) for the four Ensembles.

\section{CONCLUSIONS}

We have computed the exact density of states for the three Wigner-Dyson Ensembles as well as for Class $\mathrm{C}$ and Class D by evaluating finite dimensional supersymmetric integrals analytically. In this way the $N \rightarrow \infty$-limit implicit in most of the previous calculations could be avoided. The exact results were then employed to test a $1 / N$-expansion, which differs from the usual one because it proceeds only in the bosonic sector whereas the fermionic sector is evaluated exactly. The $1 / N$-expansion therefore does not start from a supersymmetric saddle-point or saddle-point manifold. It turned out that for all ensembles only a discrete set of saddle-points is important, whereas the saddle-point manifold at $x=0$ appearing in Class $\mathrm{C} / \mathrm{D}$ is not needed for an almost exact computation of the DOS everywhere in the spectrum (including $x=0$ ) except the band edge. The $1 / N$-expansion revealed a connection between the order $\alpha$ of $N^{-1}$ multiplying the oscillatory term of the DOS and the universal short distance exponent $\beta$ of the two-level correlation function, eq. (59). In this way we obtain information about the two-point function from the one-point function. We believe that relation (59) is always fulfilled, if the spectrum is approximately "translational invariant", i.e. $R_{2}\left(x_{1}, x_{2}\right)$ depends only on $x_{2}-x_{1}$ (this is implicit in all calculations using an unfolding procedure). At points in the spectrum where this invariance is broken (as at $x=0$ for Class C/D) the two-point function has to be calculated itself, which can, of course, also be done with our method.

The $1 / N$-expansion of the DOS yields an unambiguous determination of the average level spacing $\Delta(E)$ in terms of $E$ and $N$ everywhere in the spectrum including $x=0$ for all ensembles. For Class $\mathrm{C} / \mathrm{D}$ we conclude that the special features of the DOS at the band center vanish in the macroscopic $N \rightarrow \infty$-limit and are of no relevance if the bandwidth is kept finite.

The other Gaussian ensembles are under current investigation. It should also be possible to extend the method to non-Gaussian matrix models, see e.g. [7], which are difficult to treat with orthogonal polynomials. Here it would be interesting to test the results obtained in the large $N$-limit using singular integral equations by comparing with the exact formulae for finite $N$ and the systematic $1 / N$-expansion.

\section{Acknowledgments}

This work was supported by the Graduiertenkolleg "Nonlinear Problems in Analysis, Geometry, and Physics" (GRK 283), financed by the German Science Foundation (DFG) and the State of Bavaria.
1 T. Guhr, A. Müller-Groeling and H.A. Weidenmüller, Phys. Rep. 299190 (1998) cond-mat/9707301

2 A. Altland and M.R. Zirnbauer, Phys.Rev.B 551142 (1997)

3 M.L. Mehta Random Matrices, 2th ed., Academic Press, Boston (1991)

${ }^{4}$ K. Efetov, Supersymmetry in Disorder and Chaos, Cambridge University Press, Cambridge (1997)
${ }^{5}$ T. Nagao and K. Slevin, J.Math.Phys. 34 (1993) 2317

${ }^{6}$ D.A. Ivanov, J.Math.Phys. 43126 (2002) condmat/0103137

G. Akeman and G. Vernizzi, New Critical Matrix Models and Generalized Universality, hep-th/0201165 